\title{
INFLUÊNCIA DE DIFERENTES FONTES DE ERRO SOBRE AS MEDIÇÕES DE ALTURAS DE ÁRVORES
}

\author{
Gilson Fernandes da Silva ${ }^{1}$, Onair Mendes de Oliveira ${ }^{2}$, Carlos Alberto Martinelli de Souza ${ }^{3}$, \\ Carlos Pedro Boechat Soares ${ }^{4}$, Ronielly Lemos ${ }^{5}$
}

(recebido: 4 de outubro de 2010; aceito: 28 de fevereiro de 2012)

\begin{abstract}
RESUMO: Conduziu-se este trabalho, com o objetivo principal de avaliar a influência de diferentes fontes de variação sobre as medições de alturas de árvores de Eucalyptus urophila $x$ Eucalyptus grandis. Hipsômetros baseados no princípio trigonométrico exigem que o mensurador fique a uma distância conhecida da árvore. Dessa forma, se essas distâncias forem pequenas ou muito grandes, podem ocorrer oscilações no momento da leitura do aparelho ou dificuldade de visualizar a árvore, introduzindo erros nas medições de alturas. Sendo assim, neste trabalho, foram medidas as alturas de um conjunto de árvores com três hipsômetros a três diferentes distâncias, por três diferentes pessoas. Os resultados encontrados foram comparados com as verdadeiras alturas totais das árvores, obtidas por meio da técnica de escalada. Após análises, os resultados indicaram que as distâncias de medição, juntamente com o hipsômetro utilizado, influenciam na obtenção da altura total.
\end{abstract}

Palavras-chave: Dendrometria, hipsômetros, princípios trigonométricos.

\section{INFLUENCE OF DIFFERENT SOURCES OF ERRORS ON THE MEASUREMENTS OF HEIGHTS OF TREES}

\begin{abstract}
The objective of this study was to evaluate the influence of different sources of variation in tree height measurements using hypsometers based on trigonometric principles. The use of this type of device requires that the measurement is at a known distance from the tree. Therefore, if these distances are small or too big, oscillations can occur during the reading of the device or difficulties to visualize the tree tops, causing errors in the height measurement. Thereby, in this study a set of tree heights were measured with a hypsometer at different distances and by three different individuals. Afterwards, the results were compared with the actual heights of the trees obtained by climbing them. Finally, after the analysis, the results indicated that the distance of measurement, along with the hypsometer used, affects in obtaining estimation of the total height.
\end{abstract}

Key words: Dendrometry, hipsometer, trigonometric principles.

\section{INTRODUÇÃO}

O manejo florestal moderno requer o conhecimento da floresta e sua interação com o meio, assim como do crescimento e dos produtos que podem ser obtidos para determinados fins (serraria, celulose, chapas, energia etc.). A avaliação florestal e a dinâmica dos povoamentos só podem ser determinadas por meio de medições de amostras representativas dessas florestas. Nesse sentido, variáveis como o DAP (diâmetro à altura do peito) e a altura total são especialmente importantes (COUTO; BASTOS, 1988; FREITAS; WICHERT, 1998).
A altura constitui-se em uma importante característica da árvore e pode ser determinada ou estimada. Em florestas plantadas, sua determinação ou estimação é muito importante para o cálculo do volume, de incrementos em altura e, em determinadas situações, pode servir como indicadora da qualidade produtiva de um local (SOARES et al., 2006). De acordo com a Food and Agriculture Organization of the United Nations - FAO (1981), apesar de o volume representar com maior precisão a qualidade produtiva, a altura dominante é bastante empregada, pela facilidade de determinação e por ser pouco influenciada pelo espaçamento e desbastes realizados.

\footnotetext{
${ }^{1}$ Engenheiro Florestal, Professor Doutor em Ciência Florestal - Universidade Federal do Espírito Santo - Departamento de Engenharia Florestal Cx. P.16 - 29500-000 - Alegre, ES, Brasil - gfsilva2000@yahoo.com

${ }^{2}$ Engenheiro Florestal, Mestrando em Ciências Florestais - Universidade Federal do Espírito Santo - Departamento de Engenharia Florestal Cx. P.16 - 29500-000 - Alegre, ES, Brasil - onairmendes@yahoo.com.br

${ }^{3}$ Engenheiro Florestal, Professor Doutor em Engenharia Florestal - Universidade Federal de São Carlos - Campus Sorocaba - Rodovia João Leme dos Santos, Km 110 - 18052-780 - Sorocaba, SP, Brasil - camartinelli5@hotmail.com

${ }^{4}$ Engenheiro Florestal, Professor Doutor em Ciência Florestal - Universidade Federal de Viçosa - Departamento de Engenharia Florestal - 36571-000 Viçosa, MG, Brasil - csoares@ufv.br

${ }^{5}$ Graduando em Engenharia Florestal - Universidade Federal do Espírito Santo - Departamento de Engenharia Florestal - Cx. P. 16 - 29500-000 Alegre, ES, Brasil - ronilemos@hotmail.com
}

Cerne, Lavras, v. 18, n. 3, p. 397-405, jul./set. 2012 
Além disso, o conhecimento da altura é fundamental para a definição de estratégias que buscam otimizar o uso do fuste das árvores para multiprodutos, como serraria, celulose e energia.

Em florestas nativas, a altura total das árvores pode ter importante significado ecológico, à medida que ajuda a compreender a estrutura vertical da comunidade, podendose estimar, por meio dessa variável, as espécies que apresentam maior importância ecológica considerando-se esse tipo de estrutura. A posição sociológica das espécies pode ser calculada tomando-se em conta as alturas das árvores amostradas na comunidade, de acordo, por exemplo, com metodologias apresentadas por Finol (1971), Longhi (1980) e Souza e Leite (1993).

De acordo com Machado e Figueiredo Filho (2003), a altura total de uma árvore pode ser definida como a distância do nível do solo até o topo da árvore, ao longo de seu eixo principal. Diferentemente do diâmetro, a medição da altura não é tarefa tão fácil. No entanto, existem diversos tipos de aparelhos que podem ser utilizados para esse fim, sendo mais utilizados no meio florestal os hipsômetros (GONÇALVES et al., 2009). O uso de um hipsômetro possui aspectos positivos e negativos. Nesse sentido, a escolha de um em detrimento dos demais depende da situação e finalidade de uso de cada aparelho (FORESTRY RESEARCH WORKING GROUP 2, 1999).

De acordo com Brack (2008), alguns hipsômetros são baseados em princípios trigonométricos, tais como: Nível de Abney, Haga, Suunto Clinômetro e BlumeLeiss. Há ainda o hipsômetro Vertex Forestor que é um tipo de hipsômetro eletrônico que se utiliza de ondas sonoras e que também é baseado no princípio trigonométrico.

Para Couto e Bastos (1988), a densidade do povoamento pode influenciar a medição da altura das árvores, pois esta impossibilita a determinação de um ponto ótimo de visão no topo da árvore. Porém, Lingnau et al. (2008) afirmam que esse tipo de problema é mais frequente em florestas nativas, pois em florestas plantadas com espaçamentos regulares, o correto posicionamento do mensurador em relação à linha de plantio diminuem esse tipo de problema.

Em florestas plantadas homogêneas com espaçamentos regulares, normalmente se medem as alturas de algumas árvores com hipsômetros óticos baseados em princípios trigonométricos e a partir da composição de uma amostra que contenha os diâmetros e alturas das árvores, ajustam-se relações hipsométricas que permitirão estimar as alturas das demais árvores. Esse procedimento é muito empregado nesse tipo de florestas, como já mencionado, porque apresenta boa precisão e diminui o esforço na obtenção das alturas em campo, o que é interessante, pois estas apresentam difícil obtenção (LINGNAU et al., 2008).

Para que as relações hipsométricas produzam estimativas mais confiáveis, é importante que as medidas de altura realizadas com aparelhos óticos apresentem os menores erros possíveis. Considerando os aparelhos baseados em princípios trigonométricos, fatores como a distância do mensurador à árvore, tipo de aparelho, o nível de treinamento do operador e a própria altura total da árvore podem afetar a precisão das medidas realizadas. Assim, Campos e Leite (2006) sugerem que uma distância adequada para se realizar medições de altura seria aquela equivalente a altura aproximada da árvore. Entretanto, praticamente inexistem trabalhos científicos que procuram demonstrar a influência desses fatores na realização de medidas de altura.

Considerando o exposto, neste trabalho, objetivouse avaliar a influência dos fatores distância do mensurador à árvore, tipo de aparelho, classe de altura e diferentes operadores na medição da altura de árvores, empregandose aparelhos construídos com base em princípios trigonométricos.

\section{MATERIAL E MÉTODOS}

\subsection{Coleta de dados e modelo experimental}

O trabalho foi desenvolvido em um plantio homogêneo com o híbrido obtido a partir do Eucalyptus urophylla e E.grandis, denominado "Eucalipto urograndis". O plantio está localizado no município de Guaçuí, região sul do Espírito Santo, a 622 metros de altitude, com coordenadas centrais aproximadas no Sistema de Projeção Cartográfica UTM, Datum SAD 69, Zona 24S iguais a: $220.051 \mathrm{~m}$ e $7.690 .288 \mathrm{~m}$.

Para os testes, foram utilizados o clinômetro digital Häglof (aparelho 1), o hipsômetro Haga (aparelho 2) e o hipsômetro Vertex III (aparelho 3). O hipsômetro Haga permite realizar medidas de altura das árvores nas distâncias fixas de: 15, 20, 25 e 30 metros, enquanto que o clinômetro digital Häglof e o hipsômetro Vertex III permitem realizar leituras a qualquer distância, desde que estas sejam conhecidas.

Para avaliar o erro cometido na estimação da altura total com hipsômetros baseados em princípios trigonométricos, foi proposto um arranjo fatorial considerando-se três fatores que poderiam influenciar a ocorrência de erros no processo de medição, a saber: $\mathrm{O}$ tipo de aparelho (clinômetro digital, Haga, Vertex); a distância

Cerne, Lavras, v. 18, n. 3, p. 397-405, jul./set. 2012 
reduzida entre o mensurador e a árvore $(15,20,25$ e 30 metros), obtida com trena; e a altura total da árvore. No caso da altura total, foram consideradas três classes de altura: árvores com até 15 metros (Classe 1), entre 15-25 metros (Classe 2), e maiores que 25 metros (Classe 3).

Os dados foram coletados seguindo-se os seguintes passos:

1) Considerando-se as três classes de altura anteriormente definidas, foram selecionadas aleatoriamente no interior do plantio, 15 árvores em cada classe de altura, totalizando 45 árvores. Cada árvore foi plaqueteada e devidamente identificada.

2) As árvores selecionadas tiveram as suas alturas totais medidas com uma trena, em procedimento de escalada. Essa altura passou a ser considerada como a altura verdadeira da árvore $(H)$.

3) Em seguida, sem conhecer a verdadeira altura total das árvores, três mensuradores treinados mediram as alturas das árvores $(\hat{H})$ com cada hipsômetro e em cada distância. As medidas com cada aparelho e em cada distância foram feitas em momentos diferentes para se evitar influências na obtenção das medições. Como exemplo, o mensurador 1 empregando o aparelho 1 , mediu a altura total de todas as árvores na distância de 15 metros, repetindo esse processo para todas as demais combinações. Tomou-se o cuidado também de que os três mensuradores não tivessem contato no momento das medições. A utilização de três mensuradores justificou-se para se avaliar o efeito humano no processo da medição.

Os resultados obtidos foram avaliados calculandose o módulo do erro relativo para todas as combinações de aparelho, classe, distância e mensurador, tomando como base os valores reais de altura das árvores e as estimativas obtidas com os hipsômetros, tal como se segue:

$$
E R(\%)=\frac{|H-\hat{H}|}{H} 100
$$

em que:

$E R(\%)=$ Erro relativo, em percentagem;

$H_{i}=$ Altura real da árvore, em metros;

$\hat{H}=$ Altura estimada com os hipsômetros avaliados, em metros.

Considerando o modelo fatorial anteriormente descrito, realizou-se a análise de variância para cada mensurador, testando-se as hipóteses pelo teste $F$ em nível de 5\% de significância. Quando necessário, foram realizados testes de médias empregando-se o teste Tukey, também em nível de 5\% de significância.

\subsection{Estatísticas de comparação}

Com o intuito de complementar a análise de variância proposta no item 3.1, foram calculadas as estatísticas viés $(V)$, média das diferenças absolutas $(M D)$ e desvio-padrão das diferenças ( $D P D)$, tal como utilizado por Assis (2000), Ferreira (1999), Lima (1986), Queiroz et al. (2006), Schneider et al. (2009), Scolforo et al. (1998) e Souza et al. (2008), entre outros pesquisadores.

De acordo com os autores citados, essas estatísticas podem ser calculadas pelas seguintes expressões:

$$
V=\frac{\sum_{i=1}^{n} Y_{i}-\sum_{i=1}^{n} \hat{Y}_{i}}{n}
$$

$$
M D=\frac{\sum_{i=1}^{n}\left|Y-\hat{Y}_{i}\right|}{n} \mathrm{e}
$$

$D P D=\sqrt{\left(\sum_{i=1}^{n} d_{i}{ }^{2}-\left(\sum_{i=1}^{n} d_{i}\right)^{2} / n\right) / n}$.

Admitindo-se que cada combinação entre aparelho, distância e classe de altura representa um tratamento, a partir do cálculo das estatísticas $(V),(M D)$ e $(D P D)$, foi possível ordenar os tratamentos que apresentaram melhor desempenho na estimação da altura total das árvores. Para tanto, as estatísticas $(V),(M D)$ e ( $D P D)$ foram avaliadas, sendo atribuídos pesos de 1 a 36 para cada tratamento analisado, para cada operador. Dessa forma, sob a ótica dessa análise, os tratamentos que apresentaram a menor soma dos pesos atribuídos aos valores de cada estatística, proporcionaram melhor desempenho na estimação da altura total das árvores. Além disso, foram construídos gráficos com os valores dos resíduos de modo a fundamentar as conclusões baseadas nessas estatísticas.

\section{RESULTADOS E DISCUSSÃO}

\subsection{Análise de variância do erro relativo para cada mensurador}

Observa-se, na Tabela 1, que, para todos os mensuradores avaliados, a interação tripla (aparelho $x$ distância $x$ classe) não foi significativa pelo teste $F$, em nível de $5 \%$ de probabilidade. Por outro lado, para o mensurador 1, houve uma interação dupla entre aparelho e classe, e também diferenças entre as médias para o fator distância. Com relação aos mensuradores 2 e 3, as

Cerne, Lavras, v. 18, n. 3, p. 397-405, jul./set. 2012 
únicas diferenças detectadas foram entre as médias do fator classe. Esses resultados inicialmente sugerem que mensuradores com diferentes níveis de experiência podem produzir diferentes níveis de erros no uso de aparelhos. Embora os três operadores tenham recebido o mesmo treinamento prévio para a execução da pesquisa, eles possuem habilidades e experiências anteriores diferentes e não exercem este tipo de tarefa cotidianamente.

$\mathrm{O}$ treinamento adequado e a prática intensiva podem minimizar as diferenças entre os mensuradores, embora, nesse trabalho, elas não pareçam tão elevadas. Não se pode descartar também o fato de que as condições do ambiente podem provocar diferenças entre as medidas realizadas pelos diferentes mensuradores. Condições de luz, presença de chuva, entre outros, podem ser fontes aleatórias de erros. Esses fatores não parecem ter sido significativos nesta pesquisa, uma vez que as medidas foram feitas em um curto espaço de tempo e com condições favoráveis do ambiente.

Na Tabela 2, apresentam-se os resultados dos testes de médias, empregando-se a estatística de Tukey, em nível de $5 \%$ de significância.
Tabela 1 -Análise de variância para o erro relativo, considerandose os fatores classe, distância e aparelho.

Table 1-Analysis of variance for the relative error, considering the class factors, distance and device.

\begin{tabular}{lcccc}
\hline & & \multicolumn{3}{c}{ Operadores } \\
\cline { 3 - 5 } Fontes de Variação & GL & 1 & 2 & 3 \\
\cline { 3 - 5 } & & $F$ calc. & $F$ calc. & $F$ calc. \\
\hline Classe & 2 & $15,24^{* *}$ & $3,41^{*}$ & $6,71^{* *}$ \\
Distância & 3 & $5,95^{* *}$ & $1,85^{\text {ns }}$ & $1,20^{\text {ns }}$ \\
Aparelho & 2 & $7,55^{* *}$ & $0,39^{\text {ns }}$ & $1,27^{\text {ns }}$ \\
Distância $x$ Classe & 6 & $1,97^{\text {ns }}$ & $0,98^{\text {ns }}$ & $1,18^{\text {ns }}$ \\
Aparelho $x$ Classe & 4 & $2,46^{*}$ & $0,73^{\text {ns }}$ & $0,39^{\text {ns }}$ \\
Aparelho $x$ Distância & 6 & $1,12^{\text {ns }}$ & $0,28^{\text {ns }}$ & $0,84^{\text {ns }}$ \\
Aparelho $x$ Distância $x$ Classe & 12 & $0,67^{\text {ns }}$ & $0,38^{\text {ns }}$ & $1,02^{\text {ns }}$ \\
Resíduo & 504 & - & - & - \\
\hline
\end{tabular}

* e ** representam significância a $5 \%$ e a $1 \%$ de probabilidade pelo teste $F$, respectivamente; nsindica que é não significativo a $5 \%$ de probabilidade.

* and ** represent significance at $5 \%$ and $1 \%$ probability by $F$ test, respectively; ns indicates that it is not significant at $5 \%$ probability.

Tabela 2 - Comparações entre médias de tratamentos, pelo teste de Tukey a 5\% de significância, para o desdobramento da interação significativa (Aparelho*Classe) e fator distância para o mensurador 1, e do fator Classe para os mensuradores 2 e 3.

Table 2 - Comparisons between treatment means by Tukey test at 5\% significance for the unfolding of interaction (hypsometer*Class), and distance factor of measurement one, and class factor of measurements 2 and 3.

\begin{tabular}{|c|c|c|c|c|c|}
\hline \multicolumn{6}{|c|}{ Mensurador 1 - Testes de médias do desdobramento aparelho x classe } \\
\hline \multicolumn{2}{|c|}{ Clinômetro } & \multicolumn{2}{|c|}{ Haga } & \multicolumn{2}{|c|}{ Vertex III } \\
\hline Classe & Média (\%) & Classe & Média (\%) & Classe & Média $(\%)$ \\
\hline 3 & $5,4980 \mathrm{a}$ & 3 & $4,3620 \mathrm{a}$ & 3 & $5,2157 \mathrm{a}$ \\
\hline 1 & $4,6532 \mathrm{ab}$ & 2 & 3,4823 a & 2 & $2,9128 \mathrm{~b}$ \\
\hline 2 & $3,9098 \mathrm{~b}$ & 1 & $3,2839 \mathrm{a}$ & 1 & $2,1555 \mathrm{~b}$ \\
\hline \multicolumn{2}{|c|}{ Classe 1} & \multicolumn{2}{|c|}{ Classe 2} & \multicolumn{2}{|c|}{ Classe 3} \\
\hline Aparelho & Média (\%) & Aparelho & Média (\%) & Aparelho & Média (\%) \\
\hline Clinômetro & $4,6532 \mathrm{a}$ & Clinômetro & 3,9098 a & Clinômetro & $4,6771 \mathrm{a}$ \\
\hline Haga & $3,4823 \mathrm{ab}$ & Haga & $3,2839 \mathrm{a}$ & Haga & $3,7094 \mathrm{~b}$ \\
\hline VertexIII & $2,1555 \mathrm{~b}$ & VertexIII & $2,9128 \mathrm{a}$ & VertexIII & $3,4280 \mathrm{~b}$ \\
\hline \multicolumn{2}{|c|}{ Mensurador 1} & \multicolumn{2}{|c|}{ Mensurador 2} & \multicolumn{2}{|c|}{ Mensurador 3} \\
\hline Distância (m) & Média (\%) & Classe & Média (\%) & Classe & Média (\%) \\
\hline 15 & $4,9104 \mathrm{a}$ & 3 & $5,2293 \mathrm{a}$ & 3 & $5,2629 \mathrm{a}$ \\
\hline 30 & $3,9060 \mathrm{ab}$ & 2 & $4,7656 a b$ & 2 & $3,6875 \mathrm{~b}$ \\
\hline 25 & $3,4890 \mathrm{~b}$ & 1 & $4,0393 \mathrm{~b}$ & 1 & $3,4475 \mathrm{~b}$ \\
\hline 20 & $3,4606 \mathrm{~b}$ & - & - & - & - \\
\hline
\end{tabular}

Médias seguidas pela mesma letra não diferem estatisticamente.

Means followed by same letter do not differ statistically.

Cerne, Lavras, v. 18, n. 3, p. 397-405, jul./set. 2012 
Primeiramente, são analisados os resultados para o mensurador 1, em relação ao desdobramento da interação entre aparelho e classe. Considerando-se as médias das classes dentro de cada aparelho, nota-se que para os aparelhos digitais (clinômetro e Vertex) os erros foram maiores para a classe 3, diferentemente do Haga, que apresentou a mesma média de erro para as três classes avaliadas, em nível de $5 \%$ de significância pelo teste Tukey. Na classe 3, apresentam-se as maiores árvores, exigindo, portanto, uma maior inclinação do hipsômetro para se realizar as medidas de altura total.

Pode-se supor, então, que os aparelhos digitais foram mais sensíveis a essa inclinação, o que pode ter provocado esse maior erro para essa classe. De fato, no aparelho Haga, em razão do seu tamanho, é mais fácil controlar essa inclinação. O oposto ocorre com o clinômetro digital Hagloff, que, em função de seu tamanho reduzido, é mais difícil controlar pequenas oscilações.

Avaliando o efeito da distância na ocorrência dos erros para o mensurador 1 (Tabela 2), percebe-se que os maiores erros foram encontrados para a menor distância (15 metros) e os melhores resultados podem ser atribuídos às distâncias intermediárias de 20 e 25 metros. Esses resultados estão de acordo com o que se espera, uma vez que, ao se aproximar mais da árvore, é necessário uma maior inclinação do aparelho. Essa maior inclinação, como discutido anteriormente, pode levar a maiores erros. Afastar-se muito da árvore também pode não ser a melhor alternativa, uma vez que a visão da copa para a definição da altura total pode ficar prejudicada. Resultados semelhantes foram encontrados por Couto e Bastos (1988), em que o menor erro percentual foi obtido para a distância de 20 metros da árvore.
Finalmente, quando se avalia a ocorrência de erros para os mensuradores 2 e 3, o único fator estatisticamente influente foi a classe de altura. Pela observação da Tabela 2, percebe-se que os erros foram nitidamente maiores para a classe 3 de altura, ou seja, para árvores maiores do que 25 metros de altura total. Esses resultados corroboram com os obtidos para o mensurador 1 , ou seja, é mais difícil medir árvores mais altas pelo simples fato de que é mais difícil ver a copa e também se tem que inclinar mais o aparelho. Essas informações podem ser úteis para o treinamento de equipes de inventários, especialmente de povoamentos mais velhos e em locais mais produtivos, onde a chance de encontrar árvores mais altas é maior.

Embora esse trabalho tenha verificado algo que se esperava, a sua importância está na quantificação da magnitude dos erros face aos fatores que afetam a medição das alturas. Os erros encontrados, em média, mesmo nas situações mais adversas, excederam em muito pouco a casa dos 5\%, indicando a precisão dos métodos de estimação de altura avaliados. Por outro lado, é necessário avaliar a variabilidade desses erros e eventuais tendências na sua ocorrência, o que é feito na sequência.

\subsection{Análise das estatísticas viés (V), médias das diferenças absolutas (MD) e desvio padrão das diferenças (DPD)}

Avaliando a soma dos pesos atribuídos a cada estimativa encontrada para as estatísticas V, MD e DPD, para cada combinação entre o fator distância e o fator classe, percebe-se claramente que, para uma mesma distância de medição, a referida soma é maior para as maiores classes de altura (Tabela 3). Valores altos dessa soma evidenciam que as estatísticas do tratamento em questão apresentam piores resultados na estimação da altura quando comparados com outros tratamentos.

Tabela 3 - Resultado das estatísticas viés (V), média das diferenças absolutas (MD) e desvio padrão das diferenças (DPD) e ranking para cada tratamento avaliado separados por operador.

Table 3 - Results of statistics bias (V), mean of absolute differences (MD) and standard deviation of differences (DPD) and ranking for each treatment evaluated separately by operator.

\begin{tabular}{cccccccccccc}
\hline & \multicolumn{10}{c}{ Operador 1 } \\
\hline TRAT & $\mathrm{V}$ & $\mathrm{MD}$ & $\mathrm{DPD}$ & $\mathrm{T}$ & $\mathrm{P}$ & $\mathrm{TRAT}$ & $\mathrm{V}$ & $\mathrm{MD}$ & $\mathrm{DPD}$ & $\mathrm{T}$ & $\mathrm{P}$ \\
\hline C1D15A1 & 0,280 & 0,387 & 0,361 & 47 & 16 & C1D20A1 & $-0,040$ & 0,307 & 0,490 & 27 & 8 \\
C1D15A2 & 0,093 & 0,280 & 0,315 & 22 & 5 & C1D20A2 & 0,160 & 0,333 & 0,419 & 41 & 13 \\
C1D15A3 & $\mathbf{- 0 , 0 4 7}$ & $\mathbf{0 , 1 5 3}$ & $\mathbf{0 , 2 3 3}$ & $\mathbf{1 1}$ & $\mathbf{1}$ & C1D20A3 & $-0,133$ & 0,160 & 0,239 & 24 & 7 \\
\hline & & & & & & & & & To be continued...
\end{tabular}

Cerne, Lavras, v. 18, n. 3, p. 397-405, jul./set. 2012 
Tabela 3 - Continuação...

Table 3 - Continued...

\begin{tabular}{|c|c|c|c|c|c|c|c|c|c|c|c|}
\hline TRAT & V & MD & DPD & $\mathrm{T}$ & $\mathrm{P}$ & TRAT & V & MD & DPD & $\mathrm{T}$ & $\mathrm{P}$ \\
\hline C1D25A1 & $-0,187$ & 0,387 & 0,394 & 42 & 14 & $\mathrm{C} 1 \mathrm{D} 30 \mathrm{~A} 1$ & $-0,273$ & 0,420 & 0,395 & 49 & 17 \\
\hline C1D25A2 & 0,020 & 0,287 & 0,353 & 17 & 3 & $\mathrm{C} 1 \mathrm{D} 30 \mathrm{~A} 2$ & 0,007 & 0,233 & 0,317 & 12 & 2 \\
\hline C1D25A3 & $-0,120$ & 0,213 & 0,234 & 22 & 6 & C1D30A3 & $-0,127$ & 0,167 & 0,231 & 20 & 4 \\
\hline C2D15A1 & $-0,267$ & 1,040 & 1,327 & 73 & 26 & $\mathrm{C} 2 \mathrm{D} 20 \mathrm{~A} 1$ & $-0,407$ & 0,593 & 0,602 & 66 & 22 \\
\hline C2D15A2 & $-0,253$ & 0,880 & 1,036 & 70 & 24 & $\mathrm{C} 2 \mathrm{D} 20 \mathrm{~A} 2$ & $-0,133$ & 0,560 & 0,660 & 51 & 18 \\
\hline C2D15A3 & $-0,140$ & 0,727 & 0,876 & 62 & 21 & $\mathrm{C} 2 \mathrm{D} 20 \mathrm{~A} 3$ & 0,093 & 0,440 & 0,542 & 40 & 11 \\
\hline C2D25A1 & 0,020 & 0,527 & 0,714 & 42 & 10 & $\mathrm{C} 2 \mathrm{D} 30 \mathrm{~A} 1$ & $-0,113$ & 0,780 & 0,946 & 58 & 20 \\
\hline $\mathrm{C} 2 \mathrm{D} 25 \mathrm{~A} 2$ & 0,040 & 0,360 & 0,433 & 30 & 9 & $\mathrm{C} 2 \mathrm{D} 30 \mathrm{~A} 2$ & $-0,340$ & 0,700 & 0,805 & 71 & 25 \\
\hline C2D25A3 & 0,060 & 0,460 & 0,627 & 33 & 12 & $\mathrm{C} 2 \mathrm{D} 30 \mathrm{~A} 3$ & 0,047 & 0,593 & 0,781 & 44 & 15 \\
\hline C3D15A1 & 2,793 & 2,967 & 2,662 & 108 & 36 & $\mathrm{C} 3 \mathrm{D} 20 \mathrm{~A} 1$ & 0,020 & 1,247 & 1,575 & 53 & 19 \\
\hline $\mathrm{C} 3 \mathrm{D} 15 \mathrm{~A} 2$ & $-0,107$ & 1,480 & 2,003 & 78 & 28 & $\mathrm{C} 3 \mathrm{D} 20 \mathrm{~A} 2$ & $-0,133$ & 1,227 & 1,779 & 73 & 27 \\
\hline C3D15A3 & $-1,533$ & 1,960 & 1,893 & 103 & 35 & $\mathrm{C} 3 \mathrm{D} 20 \mathrm{~A} 3$ & $-0,853$ & 1,640 & 1,806 & 99 & 34 \\
\hline $\mathrm{C} 3 \mathrm{D} 25 \mathrm{~A} 1$ & $-0,087$ & 1,433 & 1,774 & 68 & 23 & $\mathrm{C} 3 \mathrm{D} 30 \mathrm{~A} 1$ & $-0,200$ & 1,480 & 1,790 & 85 & 30 \\
\hline $\mathrm{C} 3 \mathrm{D} 25 \mathrm{~A} 2$ & $-0,313$ & 1,367 & 1,816 & 91 & 32 & $\mathrm{C} 3 \mathrm{D} 30 \mathrm{~A} 2$ & $-0,287$ & 1,353 & 1,710 & 82 & 29 \\
\hline C3D25A3 & $-0,507$ & 1,293 & 1,730 & 87 & 31 & $\mathrm{C} 3 \mathrm{D} 30 \mathrm{~A} 3$ & $-0,313$ & 1,607 & 2,165 & 97 & 33 \\
\hline \multicolumn{12}{|c|}{ Operador 2} \\
\hline TRAT & $\mathrm{V}$ & MD & DPD & $\mathrm{T}$ & $\mathrm{P}$ & TRAT & V & MD & DPD & $\mathrm{T}$ & $\mathrm{P}$ \\
\hline C1D15A1 & 0,000 & 0,373 & 0,458 & 10 & 3 & C1D20A1 & $-0,047$ & 0,340 & 0,476 & 13 & 4 \\
\hline C1D15A2 & 0,047 & $\mathbf{0 , 3 1 3}$ & $\mathbf{0 , 4 3 2}$ & 7 & 1 & $\mathrm{C} 1 \mathrm{D} 20 \mathrm{~A} 2$ & 0,020 & 0,367 & 0,465 & 9 & 2 \\
\hline C1D15A3 & $-0,240$ & 0,413 & 0,478 & 38 & 12 & C1D20A3 & $-0,193$ & 0,353 & 0,486 & 27 & 8 \\
\hline C1D25A1 & 0,073 & 0,407 & 0,546 & 30 & 10 & C1D30A1 & $-0,047$ & 0,367 & 0,507 & 20 & 5 \\
\hline C1D25A2 & $-0,027$ & 0,427 & 0,566 & 26 & 6 & C1D30A2 & $-0,060$ & 0,393 & 0,532 & 26 & 7 \\
\hline C1D25A3 & $-0,147$ & 0,413 & 0,568 & 38 & 13 & C1D30A3 & $-0,133$ & 0,373 & 0,538 & 29 & 9 \\
\hline $\mathrm{C} 2 \mathrm{D} 15 \mathrm{~A} 1$ & 0,420 & 0,807 & 0,893 & 64 & 21 & $\mathrm{C} 2 \mathrm{D} 20 \mathrm{~A} 1$ & 0,207 & 0,753 & 0,899 & 56 & 19 \\
\hline $\mathrm{C} 2 \mathrm{D} 15 \mathrm{~A} 2$ & $-0,153$ & 1,060 & 1,178 & 64 & 22 & $\mathrm{C} 2 \mathrm{D} 20 \mathrm{~A} 2$ & 0,047 & 0,727 & 0,852 & 41 & 14 \\
\hline C2D15A3 & $-0,327$ & 0,900 & 1,076 & 71 & 25 & $\mathrm{C} 2 \mathrm{D} 20 \mathrm{~A} 3$ & $-0,040$ & 0,693 & 0,911 & 37 & 11 \\
\hline $\mathrm{C} 2 \mathrm{D} 25 \mathrm{~A} 1$ & 0,127 & 0,687 & 0,917 & 45 & 16 & $\mathrm{C} 2 \mathrm{D} 30 \mathrm{~A} 1$ & 0,233 & 0,607 & 0,702 & 48 & 17 \\
\hline $\mathrm{C} 2 \mathrm{D} 25 \mathrm{~A} 2$ & 0,227 & 0,733 & 1,040 & 62 & 20 & $\mathrm{C} 2 \mathrm{D} 30 \mathrm{~A} 2$ & 0,327 & 0,887 & 1,040 & 69 & 24 \\
\hline $\mathrm{C} 2 \mathrm{D} 25 \mathrm{~A} 3$ & 0,047 & 0,687 & 0,955 & 41 & 15 & $\mathrm{C} 2 \mathrm{D} 30 \mathrm{~A} 3$ & 0,180 & 0,713 & 0,921 & 53 & 18 \\
\hline C3D15A1 & 2,513 & 2,660 & 2,683 & 107 & 36 & $\mathrm{C} 3 \mathrm{D} 20 \mathrm{~A} 1$ & $-0,533$ & 1,360 & 1,638 & 86 & 28 \\
\hline C3D15A2 & $-0,740$ & 1,807 & 2,317 & 100 & 34 & $\mathrm{C} 3 \mathrm{D} 20 \mathrm{~A} 2$ & $-0,473$ & 1,367 & 1,663 & 87 & 29 \\
\hline C3D15A3 & $-1,913$ & 2,140 & 2,071 & 102 & 35 & C3D20A3 & $-1,207$ & 1,713 & 2,034 & 98 & 32 \\
\hline $\mathrm{C} 3 \mathrm{D} 25 \mathrm{~A} 1$ & $-0,200$ & 1,320 & 1,717 & 75 & 27 & C3D30A1 & $-0,300$ & 1,267 & 1,495 & 74 & 26 \\
\hline $\mathrm{C} 3 \mathrm{D} 25 \mathrm{~A} 2$ & $-0,453$ & 1,507 & 1,957 & 91 & 30 & $\mathrm{C} 3 \mathrm{D} 30 \mathrm{~A} 2$ & $-0,100$ & 1,220 & 1,548 & 64 & 23 \\
\hline C3D25A3 & $-0,580$ & 1,407 & 1,885 & 92 & 31 & $\mathrm{C} 3 \mathrm{D} 30 \mathrm{~A} 3$ & 0,360 & 2,293 & 4,601 & 98 & 33 \\
\hline
\end{tabular}

Cerne, Lavras, v. 18, n. 3, p. 397-405, jul./set. 2012 
Tabela 3 - Continuação...

Table 3 - Continued...

\begin{tabular}{cccccccccccc}
\hline & \multicolumn{10}{c}{ Operador 3 } \\
\hline TRAT & $\mathrm{V}$ & $\mathrm{MD}$ & $\mathrm{DPD}$ & $\mathrm{T}$ & $\mathrm{P}$ & $\mathrm{TRAT}$ & $\mathrm{V}$ & $\mathrm{MD}$ & $\mathrm{DPD}$ & $\mathrm{T}$ & $\mathrm{P}$ \\
\hline C1D15A1 & $\mathbf{- 0 , 1 2 0}$ & $\mathbf{0 , 2 4 0}$ & $\mathbf{0 , 2 6 8}$ & $\mathbf{1 4}$ & $\mathbf{1}$ & $\mathrm{C} 1 \mathrm{D} 20 \mathrm{~A} 1$ & $-0,007$ & 0,327 & 0,410 & 25 & 5 \\
C1D15A2 & $-0,113$ & 0,247 & 0,283 & 17 & 2 & C1D20A2 & $-0,100$ & 0,247 & 0,309 & 17 & 3 \\
C1D15A3 & $-0,293$ & 0,307 & 0,219 & 26 & 7 & C1D20A3 & $-0,273$ & 0,300 & 0,282 & 25 & 6 \\
C1D25A1 & $-0,127$ & 0,327 & 0,406 & 33 & 11 & C1D30A1 & $-0,227$ & 0,360 & 0,390 & 38 & 14 \\
C1D25A2 & $-0,087$ & 0,273 & 0,329 & 19 & 4 & C1D30A2 & $-0,227$ & 0,307 & 0,299 & 29 & 8 \\
C1D25A3 & $-0,307$ & 0,320 & 0,274 & 30 & 9 & C1D30A3 & $-0,273$ & 0,340 & 0,317 & 35 & 13 \\
C2D15A1 & $-0,380$ & 0,900 & 1,183 & 67 & 23 & C2D20A1 & 0,167 & 0,353 & 0,440 & 38 & 15 \\
C2D15A2 & $-0,400$ & 0,840 & 0,977 & 66 & 21 & C2D20A2 & $-0,113$ & 0,407 & 0,506 & 38 & 16 \\
C2D15A3 & $-0,480$ & 0,747 & 0,866 & 66 & 22 & C2D20A3 & 0,813 & 1,413 & 3,934 & 94 & 32 \\
C2D25A1 & $-0,040$ & 0,560 & 0,698 & 42 & 18 & C2D30A1 & 0,167 & 0,500 & 0,622 & 47 & 19 \\
C2D25A2 & $-0,040$ & 0,413 & 0,504 & 33 & 12 & C2D30A2 & $-0,107$ & 0,547 & 0,700 & 47 & 20 \\
C2D25A3 & 0,000 & 0,440 & 0,616 & 32 & 10 & C2D30A3 & $-0,007$ & 0,513 & 0,629 & 38 & 17 \\
C3D15A1 & $\mathbf{2 , 9 8 7}$ & $\mathbf{3 , 0 2 7}$ & $\mathbf{2 , 3 5 6}$ & $\mathbf{1 0 7}$ & $\mathbf{3 6}$ & C3D20A1 & $-0,680$ & 1,453 & 1,784 & 82 & 27 \\
C3D15A2 & $-0,567$ & 1,647 & 1,981 & 92 & 30 & C3D20A2 & $-0,780$ & 1,607 & 1,897 & 92 & 31 \\
C3D15A3 & $-1,480$ & 1,867 & 1,967 & 102 & 35 & C3D20A3 & $-0,953$ & 1,540 & 1,876 & 94 & 33 \\
C3D25A1 & $-0,740$ & 1,513 & 1,952 & 90 & 29 & C3D30A1 & $-0,353$ & 1,340 & 1,808 & 72 & 24 \\
C3D25A2 & $-0,620$ & 1,380 & 1,852 & 81 & 26 & C3D30A2 & $-0,527$ & 1,340 & 1,702 & 74 & 25 \\
C3D25A3 & $-0,933$ & 1,787 & 2,091 & 101 & 34 & C3D30A3 & $-0,873$ & 1,447 & 1,831 & 87 & 28 \\
\hline
\end{tabular}

$\mathrm{T}=$ total e $\mathrm{P}=$ posição.

$\mathrm{O}$ tratamento C1D15A1, por exemplo, indica uma medida de altura realizada em uma árvore pertencente à classe $1(\mathrm{C} 1)$, na distância de 15 metros (D15), por meio do uso do aparelho 1 (A1).

Treatment C1D15A1, for example, indicates a measure of height held in a tree belonging to Class 1 (C1), a distance of 15 meters (D15), through the use of device 1 (A1).

Por outro lado, quando essa soma é avaliada para o fator distância dentro de cada fator classe, percebe-se que os maiores valores ocorrem quando a medição é realizada na distância mais próxima da árvore. Dessa forma, quando a altura de uma árvore é estimada a uma distância muito próxima do mensurador à árvore, diversos fatores atuam e tornam a estimação mais passível de erros. No presente estudo, esse comportamento é verificado para todas as classes de altura, com exceção da classe 1 (árvores até 15 metros). Entretanto, esse comportamento era esperado, uma vez que seu menor tamanho possibilita uma melhor visada para medição.

Ainda, de acordo com a Tabela 3, nota-se que as estimativas da estatística viés (V), para todos os tratamentos, encontram-se próximas de zero, indicando ausência de tendência nas estimativas realizadas, exceto para as medidas realizadas às distâncias de 15 metros, nas árvores pertencentes a classe 3 (árvores maiores que $25 \mathrm{~m}$ ), conforme distribuição dos resíduos apresentada na Figura 1.

Esse resultado era esperado, pois em árvores mais altas é muito mais difícil realizar a visada para medição em distâncias mais próximas à árvore. Nessas distâncias, qualquer oscilação mínima do operador no momento da visada, pode resultar em erros muito maiores na estimação da altura total. Quando foram avaliados os valores da média das diferenças absolutas (MD), apenas os tratamentos que representam árvores da classe 1 se mostraram satisfatórios, apresentando valores próximos a zero.

Na classe 1, não há grandes variações dos valores das estatísticas para as diferentes distâncias de medição, ou seja, nessa situação a distância de medição não provoca influências negativas na estimação da altura de árvores com até 15 metros de altura.

Cerne, Lavras, v. 18, n. 3, p. 397-405, jul./set. 2012 
(c)

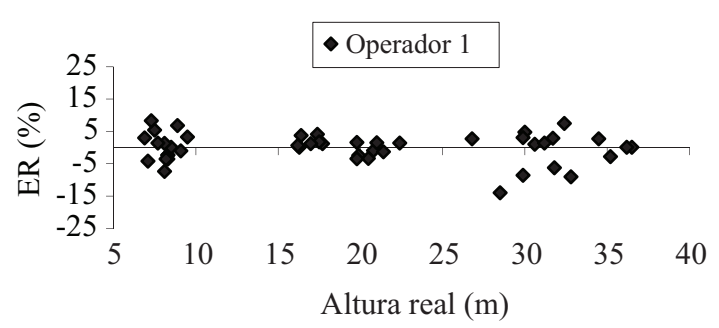

(e)

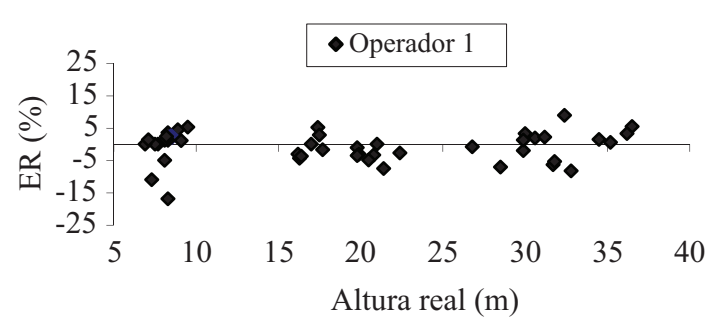

(d)

D20A3

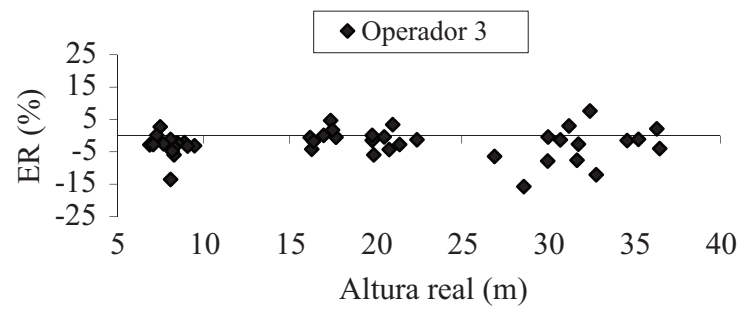

(f)

D15A1

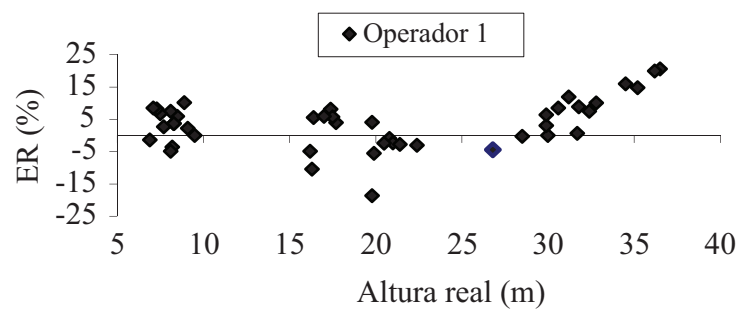

Figura 1 - Distribuição dos resíduos percentuais da altura total estimada em função da altura real, para as seguintes situações, de acordo com o ranking obtido para os tratamentos avaliados: (c) e (d), melhor e pior desempenho dentro da classe 2; (e) e (f) melhor e pior desempenho dentro da classe 3.

Figure 1 -Distribution of residue percentage of the total height estimated according to the actual height for the following situations, the ranking obtained for the treatments evaluated: (c) and (d), best and worst performance for class 2; (e) and (f) best and worst performance for class 3 .

De certa forma, como essas estatísticas demonstram o nível de precisão dos diferentes tratamentos avaliados, pode-se inferir que para árvores maiores de 15 metros, a altura deve ser estimada por meio de distâncias de medição maiores que 15 metros.

Os resultados obtidos condizem com os encontrados por Loetsch et al. (1973), que sugerem como regra prática que a melhor posição para medição, é aquela em que o operador se posiciona a uma distância no mínimo igual a altura da árvore.

\section{CONCLUSÕES}

De acordo com as condições em que foram desenvolvidos os estudos, pode-se concluir que:

- os maiores erros na medição da altura são cometidos nas árvores mais altas (classe 3), medidas a distância de 15 metros;

- de forma geral, para as árvores maiores, as distâncias de 20 e 25 metros parecem adequadas para a medição de altura;

- os aparelhos digitais apresentam um pior desempenho na medição da altura das árvores mais altas.

\section{REFERÊNCIAS}

ASSIS, A. L. Avaliação de modelos polinomiais segmentados e não segmentados na estimativa de diâmetros e volumes comerciais de Pinus taeda. 2000. 198 p. Dissertação (Mestrado em Engenharia Florestal) Universidade Federal de Lavras, Lavras, 2000.

\section{BRACK, C. Comparing diameter measuring instruments.}

Disponível em: $<$ http://online.anu.edu.au/Forestry/ mensuration/toolsd.htm>. Acesso em: 15 jun. 2008.

CAMPOS, J. C. C.; LEITE, H. G. Mensuração florestal: perguntas e respostas. 2. ed. Viçosa, MG: UFV, 2006. 470 p.

COUTO, H. T. Z.; BASTOS, N. L. M. Erros na medição de altura em povoamentos de Eucalyptusem região plana. IPEF, Piracicaba, n. 39, p. 21-31, 1988.

FERREIRA, S. O. Estudo da forma do fuste de Eucalyptus grandis e Eucalyptus cloeziana. 1999. 112 p. Dissertação (Mestrado em Ciência Florestal) - Universidade Federal de Lavras, Lavras, 1999.

Cerne, Lavras, v. 18, n. 3, p. 397-405, jul./set. 2012 
FINOL, H. Nuevos parâmetros a considerar-se en el análises estructural de las selvas virgenes tropicales. Revista Forestal Venezoelana, Merida, v. 14, n. 21, p. 24-42, 1971.

FOOD AND AGRICULTURE ORGANIZATION OF THE UNITED NATIONS. El eucalipto em la repoblación forestal. Roma, 1981. $723 \mathrm{p}$.

FORESTRY RESEARCH WORKING GROUP 2. Code of forest mensuration practice: a guide to good tree measurement practice in Australia and New Zealand. Disponível em: <http://www.anu.edu.au/Forestry/Mensuration/ rwg2/code $>$. Acesso em: 15 jun. 2008.

FREITAS, A. G. de; WICHERT, M. C. P. Comparação entre instrumentos tradicionais de medição de diâmetro e altura com o criterion 400. Piracicaba: IPEF, 1998. (Circular Técnica, 88).

GONÇALVES, D. de A.; ELDIK, T. van; POKORNY, B. Uso do dendrômetro a laser em florestas tropicais: aplicações para o manejo florestal na Amazônia. Revista Floresta, Curitiba, v. 39, n. 1, p. 175-187, 2009.

LIMA, F. Análise de funções de "taper" destinadas à avaliação de multiprodutos de árvores de Pinus elliottii. 1986. 96 f. Dissertação (Mestrado em Ciência Florestal) Universidade Federal de Viçosa, Viçosa, 1986.

LINGNAU, C.; SILVA, M.; SANTOS, D.; MACHADO, L. J. Mensuração de alturas de árvores individuais a partir de dados laser terrestre. Revista Ambiência, Guarapuava, v. 4, n. 1, p. 85-96, 2008.

LOETSCH, F. et al. Forest inventory. Munich: Verlagsgesellschaft, 1973. 469 p.
LONGHI, S. J. A estrutura de uma floresta natural de Araucaria angustifolia (Bert.) O. Ktze, no sul do Brasil. 1980. 198 f. Dissertação (Mestrado em Engenharia Florestal) Universidade Federal do Paraná, Curitiba, 1980.

MACHADO, S. do A.; FIGUEIREDO FILHO, A.

Dendrometria. Curitiba: UFPR, 2003. 309 p.

QUEIROZ, D.; MACHADO, S. A.; ARCE, J. E.; KOEHLER, H. S. Avaliação e validação de funções de afilamento para Mimosa scabrella Bentham em povoamentos da região metropolitana de Curitiba, PR. Revista Floresta, Curitiba, v. 36, n. 2, p. 46-56, 2006.

SCHNEIDER, P. R.; SCHNEIDER, P. S. P.; SOUZA, C. A. M. Análise de regressão aplicada à engenharia florestal. Santa Maria: FACOS-UFSM, 2009. 294 p.

SCOLFORO, J. R. S.; RIOS, M. S.; OLIVEIRA, A. D.; MELLO, J. M.; MAESTRI, R. Acuracidade de equações de afilamento para representar o perfil do fuste de Pinus elliotti. Cerne, Lavras, v. 4, n. 1, p. 100-122, jan./jun. 1998.

SOARES, C. P. B.; PAULA NETO, F. de; SOUZA, A. L. de. Dendrometria e inventário florestal. Viçosa, MG: UFV, 2006. 45 p.

SOUZA, A. L.; LEITE, H. G. Manejo florestal. Viçosa, MG: UFV, 1993. 147 p.

SOUZA, C. A. M.; SILVA, G. F.; XAVIER, A. C.; MENDONÇA, A. R.; ALMEIDA, A. Q. Avaliação de modelos de afilamento não-segmentados na estimação da altura e volume comercial de eucalyptussp. Ciência Florestal, Santa Maria, v. 18, n. 3, p. 141-146, 2008.

Cerne, Lavras, v. 18, n. 3, p. 397-405, jul./set. 2012 
